\title{
Effective Electromagnetic Log Data Interpretation in Realistic Reservoir Models
}

\author{
M. Epov, C. Suhorukova, V. Glinskikh, M. Nikitenko, O. Nechaev, I. Surodina \\ Institute of Petroleum Geology and Geophysics SB RAS, Novosibirsk, Russian Federation \\ Email: SuhorukovaKV@ipgg.sbras.ru
}

Received 2013

\begin{abstract}
This paper analyzes some specific features of the numerical interpretation of high-frequency electromagnetic logging data in vertical, deviated and horizontal boreholes entering oil- and water-saturated formations. The interpretation is based on numerical modeling for signals.
\end{abstract}

Keywords: High-frequency Electromagnetic Logging; VEMKZ; 2D \& 3D Modeling; Numerical Interpretation

\section{Introduction}

High-frequency electromagnetic soundings (VEMKZ) are designed for estimation of electrical resistivity distribution around borehole [1]. The system involves five or nine three-coil arrays. Phase shift (and amplitude ratio in some tools [2]) between two neighboring coils are measured. Frequencies of an exciting field are chosen in the range from $875 \mathrm{kHz}$ for a $2 \mathrm{~m}$ long probe to $14 \mathrm{MHz}$ for a $0.5 \mathrm{~m}$ long probe. A sounding curve is a combination of all probes signals at one measurement point. The curve demonstrates resistivity distribution from borehole to the uninvaded formation.

The VEMKZ method enables us to solve several problems of practical importance in inclined and horizontal boreholes: estimation of formation and invaded zone resistivities; location of a reservoir top and base as well as gas-oil and water-oil contacts position with respect to a borehole; estimation of resistivities' radial distribution from the borehole to formation in boreholes filled with high-conductive drilling mud.

In order to analyze the high-frequency electromagnetic soundings with realistic problem formulations algorithms were developed and calculation programs created, which implement the numerical analytic [3-5] and finite-difference approaches [6,7], and finite-element method [8].

\section{Electromagnetic Properties of Geological Media}

As has been observed for the decades of electrical log data numerical interpretation, every so often estimates of electrical resistivity $(\rho)$ from electromagnetic sounding data and estimates from direct current sounding (lateral logging sounding - LLS or Russian BKZ) disagree with each other.

The signals measured by various techniques were compared at intervals of thick homogeneous argillaceous deposits in marker beds, primarily, in terms of accuracy level assessment of the measuring. Signals from VEMKZ probes running at frequencies ranging from 0.875 to 14 $\mathrm{MHz}$, in such formations tend to be consistent with model containing low resistance invasion zone, whereas BKZ signals are attributed to models either with missing invasion zone, or with narrow high-resistivity zone.

The said apparent contradiction is eliminated by an extension of geoelectric model parameters, like introduction of dielectric permittivity $(\varepsilon)$ allowing to exclude invasion zone from VEMKZ data interpretation in the intervals with impermeable rocks. Figure 3 shows the result of fitting resistivity model and model including $(\rho, \varepsilon)$ parameters according to VEMKZ and BKZ data for clayey formation drilled with nonsaline clay mud.

In the course of inversion a certain effective $\varepsilon$ value was determined in which all processes of polarization in the heterogeneous medium are reflected. Consequently, with such an approach employed, $\varepsilon$ estimate is often higher, than values for each individual component composing rocks. Different researchers suggest different estimates for relative permittivity as high as several hundreds and thousands of relative units, based on the results of samples investigation (sandstone, loams, clay, [9]), and of LWD data interpretation (frequencies 0.4 and 2 $\mathrm{MHz}$, pyritized shales, [10]), with $\varepsilon$ frequency dispersion also being observed therewith.

According to VEMKZ logs, frequency dispersion of dielectric permittivity in some clayey formations is determined when the signal measured at each operating 
frequency corresponds to its own $\varepsilon$ value. Estimates of dielectric permittivity vs. frequency, obtained in several clayey formations, are consistent with the values obtained on shale samples [9].

Resistivity model is determined by BKZ data. Then such $\varepsilon$ values are matched for the obtained $\rho$ values, that calculated VEMKZ signals would fit with the measured ones. An example of such fitting in a clayey formation is shown in Figure 1. BKZ and VEMKZ measurements were simultaneous, and the well is drilled with nonsaline clay mud. BKZ signals are shown on the top in the selected resistivity model, in the middle are VEMKZ signals measured (the solid line) and calculated in resistivity model (the dotted line), at the bottom are VEMKZ signals measured and calculated in model with fitted effective value $\varepsilon$. The fitted values are $\rho=3.4 \mathrm{ohm} \cdot \mathrm{m}$, and $\varepsilon=133$ (rel.units).

The VEMKZ signals and medium dielectric permittivity relationships have been experimentally validated by the measurements performed in fresh waters of Lake Teletskoye [11]. As a result of numerical inversion of the signals recorded in the water and air-water boundary profiling, the values of electrical resistivity and relative dielectric permittivity of water $(170-190 \mathrm{ohm} \cdot \mathrm{m}$ and 62 - 67 rel. units) were obtained. The resistivity value was supported by independent measurements, and $\varepsilon$ estimate corresponds to expected value for water with similar salinity.
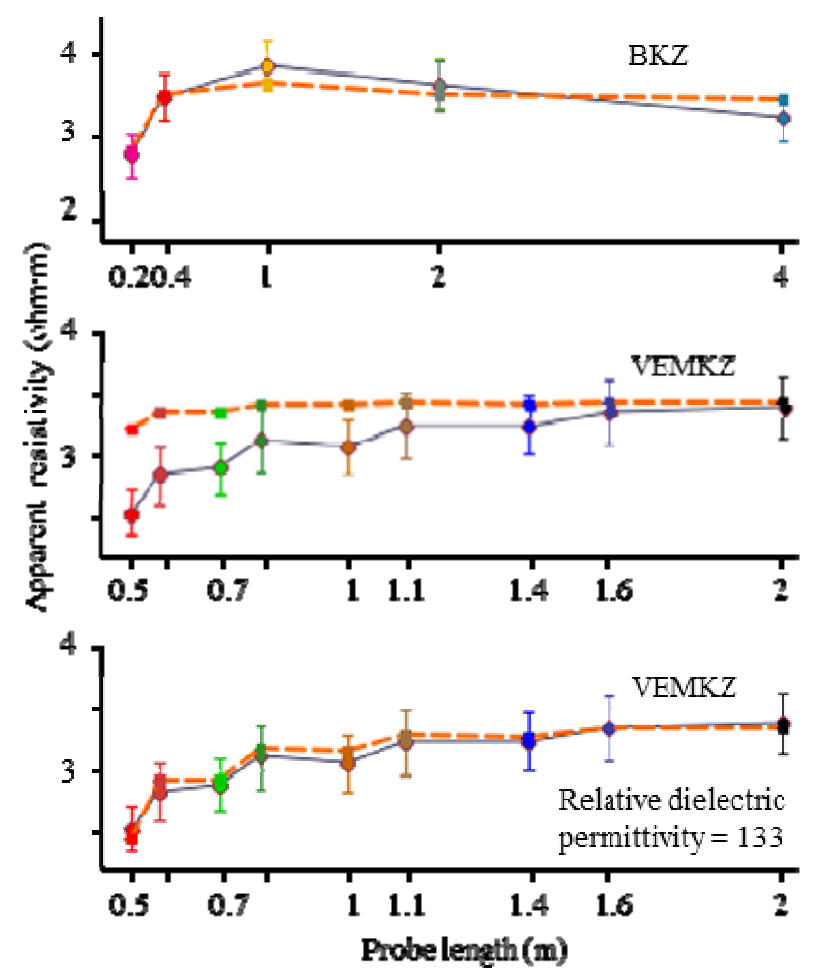

Figure 1. BKZ and VEMKZ responses measured in clayey formation.

\section{Realistic Problem Formulation and Interpretation of Model}

\subsection{Borehole Rugosity Influence}

Borehole rugosity is brought about when drilling both vertical [12], and inclined wells, in particular, using deflectors [13]. They can be represented by spiral cuttings, periodic thickening (when drilling with deflectors), as well as individual fractures, or their system. Hole rugosities, when filled with drilling mud with high electrical conductivity, cause quasiperiodic or chaotic big amplitude fluctuations (from a fraction of degree to several tens) to come up on the VEMKZ logs. The fact that changing signals account for intense rugosity of a borehole wall is supported by the correlation of caliper and VEMKZ logs.

According to the modeling results, signal fluctuations similar in form and amplitude are localized opposite shallow (some mm deep) cavities and thin fractures. The fluctuations period along the hole tend to associate with rugosity zones, and their amplitude increases with the cavity depths and operating frequency of probe, while the fluctuations pattern depends on the form of cavity and smoothness of their edges. The deviation from the level of signal in undisturbed rocks, appear to be identical on the amplitude, no matter whether it increases or decreases. This allows to exclude the influence of cavities and fractures on the signal, by its averaging.

Identical fluctuations of signals are modeled in constant diameter boreholes, however, the borehole shape can be both sinusoidal and spiral [14]. The fluctuations period is equal to the spiral period in case of spiral borehole shape, while in a sinusoidal borehole it is twice as less than the sinusoid period.

Relying on log data it is possible to evaluate rugosity parameters (Figure 2) as follows: on the basis of the fitting signals it was defined that cavity depth equals 0.007 $\mathrm{m}$, fracture depth is $1.5 \mathrm{~m}$, fracture width is $0.029 \mathrm{~m}$, fracture resistivity $0.2 \mathrm{ohm} \cdot \mathrm{m}$.

\subsection{Eccentricity Effect}

High electrical conductivity of drilling mud cause tool eccentricity to influence VEMKZ logs in the borehole [14]. As calculations have it, the bigger the borehole radius is, and the more contrasting are the electrical conductivities and the probe operating frequency, the higher is the influence. Therefore, in order to increase reliability of numerical interpretation of the data measured in wells with high electrical conductivity of drilling mud, proper correction must be made for eccentricity effect.

The algorithm, allowing to correct eccentricity effect, uses signal database calculated in the "borehole - formation" model, given the nonconducting body of the [8]. The algorithm allows to build transformation of the 
measured signal into apparent resistivity including the borehole and probe eccentricity effects, and also to calculate signals for the position on the borehole axes.

Figure 3 represents the result of algorithm applied to practical VEMKZ log data. Probe designation includes probe length in decimeters. The borehole radius is 0.062 $\mathrm{m}$, resistivity of drilling mud is $0.03 \mathrm{ohm} \cdot \mathrm{m}$. When eccentricity effect is taken into account in the clayey portion of formation, apparent resistivities for different probes become almost identical, and any noticeable divergence remains only in sandstones intervals.

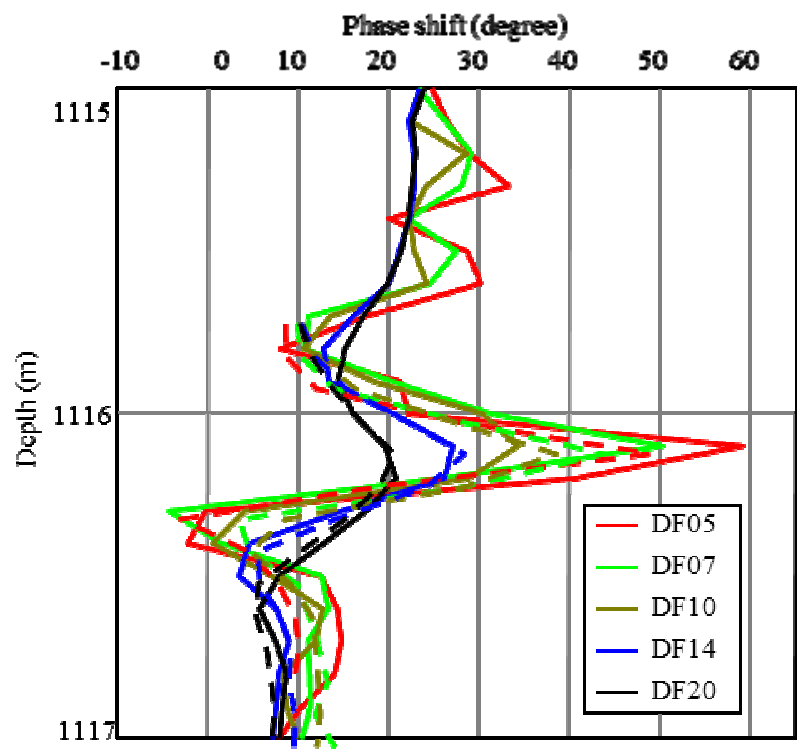

Figure 2. Measured (the solid line) and modeled (the dotted line) VEMKZ responses. Hole radius $=0.108 \mathrm{~m}$, mud resistivity $=0.1 \mathrm{ohm} \cdot \mathrm{m}$.

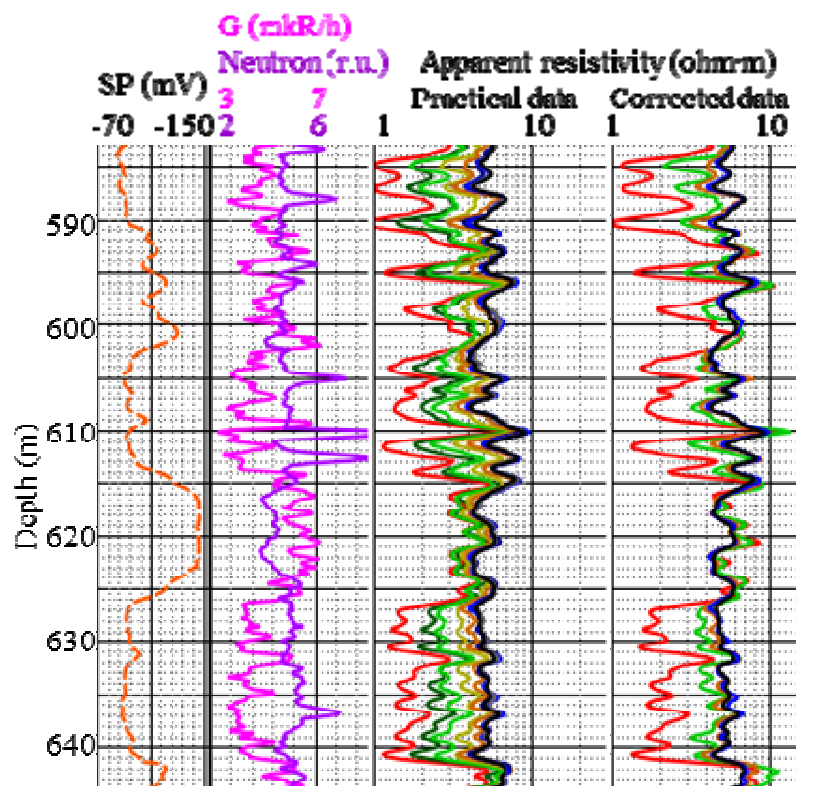

Figure 3. Measured responses and corrected responses.
With eccentricity effect taken into account, the sounding curve behavior changes, normally, in such a manner that when inversion is applied both thickness and resistivity of low-ohmic near-well zone tend to decrease. The said regularity is typical for VEMKZ logs in small-diameter boreholes with low resistive drilling mud.

\subsection{Vertical Boreholes}

Intermediate range of frequencies is used in VEMKZ, where signal is affected not only by diffusive, but also wave processes in the media. Phase shift and amplitude ratio measured in electromagnetic logging, when jointly applied, allow to reconstruct a full range of electrophysical parameters. The paper addresses the solutions of forward linear and inverse two-dimensional problems on the basis of linearized representation of relative amplitude and phase characteristics of electromagnetic field (the theory of pseudogeometrical factors) [3]. With this problem formulation, it is possible to approximate allowance for offset currents as diffusion input from wave processes, caused by $\varepsilon$ spatial distribution.

The linearized representations of electromagnetic signals measured in conducting media, given the offset currents, allow to effectively employ linear inversion in the solution of inverse problem. The solution of inverse problem includes the inversion of matrix composed of sensitivities calculated for phase shift and amplitude ratio to model parameters. Stage-by-stage approach is used for inversion. The first stage was reduced to one-dimensional inversion, which included identification of electrophysical parameters of both near-well zone and the formation, whereas during stage two they were specified $(\rho, \varepsilon)$ in the course of two-dimensional inversion.

Figure 4 presents the results of two-dimensional inversion of practical VEMKZ logs in the carbonate reservoir interval penetrated by a well with the oil-based fluids. The previously identified thin beds testify to the extent of the detailed investigation. Their thicknesses vary from 0.3 to $1.1 \mathrm{~m}$, which appears to be significantly less than the probing system length.

Vertical distributions of $\rho$ and $\varepsilon$ in the invasion zone and within formation, based on the results of one-dimensional (1) and two-dimensional (2) inversions are shown. The reliability of the results obtained can be validated in comparative analysis of the practical and calculated for the recovered model of synthetic curves for phase shifts, provided therewith. Average values of relative divergences on the considered interval account for $3 \%-4 \%$ for short probes and do not exceed $2 \%$ for long ones.

\subsection{Inclined and Horizontal Wells}

Let's consider typical model for the upper part of oil- and 
water-saturated reservoir, overlain by clayey deposits (Figure 5). The calculation has been made for horizontal beds. Clay resistivity is $4 \mathrm{ohm} \cdot \mathrm{m}$, resistivity of reservoir is $15 \mathrm{ohm} \cdot \mathrm{m}$. The borehole track (at the top of Figure 5)

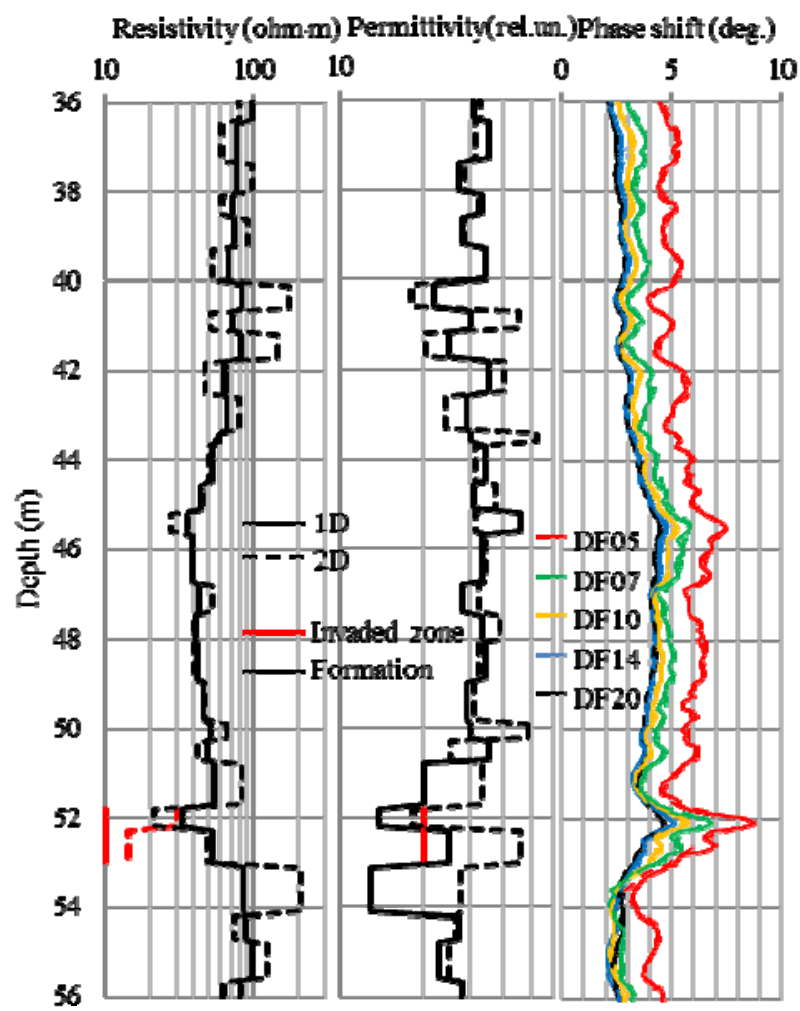

Figure 4. The results of the inversion of practical VEMKZ logs within carbonate reservoir. The recovered $1 D$ and $2 D$ distributions are $\rho$ (left) and $\varepsilon$ (middle). Practical (solid lines) and synthetic (dotted lines) curves for phase shifts (right).
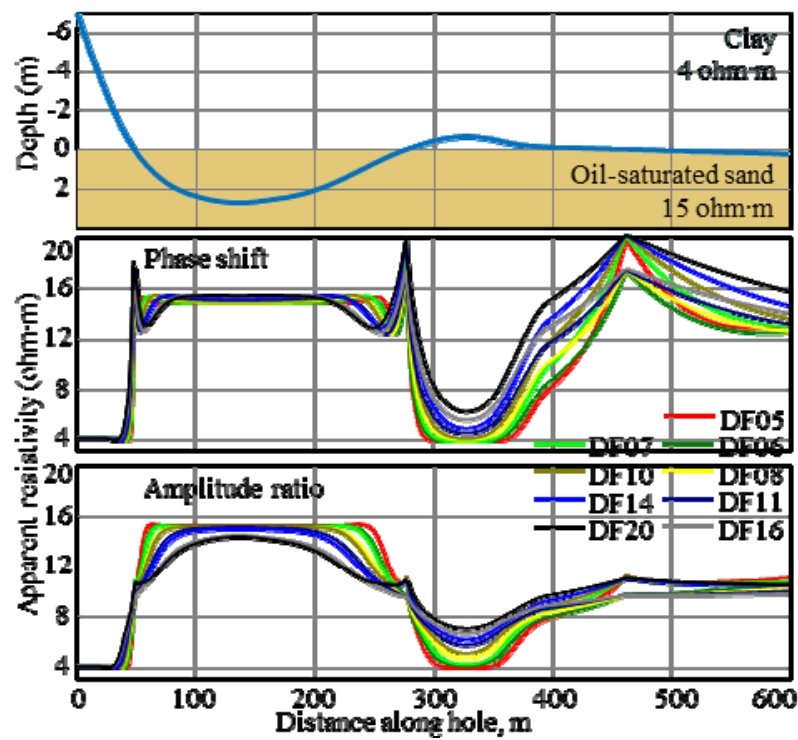

Figure 5. Model of a well with horizontal completion, penetrating the reservoir; and apparent resistivity logs. is typical in the context of West Siberia geological environment. Apparent resistivity was calculated with the use of phase shift $\left(\rho_{a}(\Delta \varphi)\right.$, at the middle) and amplitude ratio ( $\rho_{a}\left(A_{2} / A_{1}\right)$, at the bottom).

It is known that when VEMKZ tool penetrates the bed boundary there will appear electrical charges proportional to the formation resistivity contrast ratio at this boundary. The said charges affect the responses. The influence is particularly strong for the phase shift $(\Delta \varphi)$ : the phase shift diagrams show maximum peaks, which correspond to the points of boundary crossing. The more inclined is the well the higher are the spikes. Responses from the second layer appear at the intervals where distance from the boundary to the receivers is less than an offset from the transmitter to the nearest receiver ( 0.8 of probe length) the influence from, for example, another medium becomes evident, with apparent resistivity in the reservoir decreasing to $13 \mathrm{ohm} \cdot \mathrm{m}$ and increasing in the clayey bed up to $6-7 \mathrm{ohm} \cdot \mathrm{m}$. All the apparent resistivity values reach true reservoir resistivity only in the lowest part of the well $(80-200 \mathrm{~m})$, where these points are removed further than the sonde length from boundary. In a higher conductive clayey cap rock the charge response is a bit lower and apparent resistivity reaches resistivity of the clayey formation at shorter distances from the boundary, about half of sonde length. True resistivity of the clayey formation was shown by sondes with length $0.5-1.1 \mathrm{~m}$. The interval where the well extends almost along the boundary $(360-600 \mathrm{~m})$ is characterized, firstly, by difference in $\rho_{\mathrm{a}}(\Delta \varphi)$ readings from sondes with various spacing, which would mean that there is an conductive invaded zone when traditional interpretation techniques are applied; and, secondly, diagrams for the main and additional groups of probes differ. The latter could be useful in distinguishing the effects, conditioned by nearing the horizontal boundary, or by lateral inhomogeneity of reservoir properties.

Amplitude ratio is less influenced by the charge than the phase shift. The $\rho_{a}\left(A_{2} / A_{1}\right)$ logs do not show big peaks, as the boundary crossing boundaries. The logs for two deepest sondes don't reach the value of the reservoir resistivity. Sondes with lengths from 0.5 to $0.8 \mathrm{~m}$ reach the value of clay resistivity. The fact that shoulder effect is more significant for $A_{2} / A_{1}$ than it appears for $\Delta \varphi$ because the medium interval governing the amplitude ratio is bigger than the one governing the phase shift.

For water-saturated reservoirs in West Siberia resistivity values are the same as for clays. Therefore, the following reservoir model is divided into oil-water-saturated and water-saturated parts with resistivity of 15 $\mathrm{ohm} \cdot \mathrm{m}$ and $4 \mathrm{ohm} \cdot \mathrm{m}$, respectively (Figrue 6). The influence of the more conductive lower part takes place in the points located at a distance less than sonde length for $\rho_{a}(\Delta \varphi)$ and less than one and a half length for $\rho_{a}\left(A_{2} / A_{1}\right)$. In 
the interval, where the well is closer than half-length to the boundary, apparent resistivity at the $\rho_{a}(\Delta \varphi)$ curves increases and reaches maximal value at the lowest point of the well ( $0.3 \mathrm{~m}$ from boundary).

If we add a thin resistive layer to the model (usually it is carbonated sandstone with resistivity $30-100 \mathrm{ohm} \cdot \mathrm{m}$ ) it would significantly change $\rho_{\text {a }}$ near the boundary. Let's place the layer with thickness $0.2 \mathrm{~m}$ and resistivity 50 $\mathrm{ohm} \cdot \mathrm{m}$ at the boundary. In the model at Figure 5 maximum values $\rho_{a}(\Delta \varphi)$ near the boundary increase highly; under the boundary clay produces almost imperceptible lowering effect, whereas $\rho_{a}(\Delta \varphi)$ in the subhorizontal interval are significantly enhanced. At the $\rho_{a}\left(A_{2} / A_{1}\right)$ diagram maximum peaks appear in points where the well crosses the boundary. In the model shown at the Figure 6 the influence of the lower medium on the phase shift proves even smaller. At the bottom part of the well a high resistive interlayer caused an increase in $\rho_{a}$ for both parameters and changed the shape of $\rho_{a}\left(A_{2} / A_{1}\right)$ curve related to the short sonde.

In general, the increase in resistivity of high-resistance interlayer leads to the enhanced resistivity contrast and its greater growth whith the tool crossing boundaries, and to lesser dependence on the medium at the other side of the interlayer. We should note that the interlayer with these parameters is not reflected in the signals of induction logging in vertical wells.

The calculations attest to the fact that the VEMKZ signals in deviated and horizontal wells depend neither on enclosing rocks nor contrasting interlayers in the beds thicker than double probe length. In the middle part of the layer of this kind its true resistivity will be shown by $\rho_{a}(\Delta \varphi)$ diagrams for all the probes and $\rho_{a}\left(A_{2} / A_{1}\right)$ diagrams, but not including long-spaced probes. However, characteristic behavior of signals near the boundaries and singlevalued dependence on the contrast of electrical properties allows to estimate formation parameters, using program for modeling responses of the deviated tool, in case the well track is known.

Calculations of a signal in realistic model (including borehole) shows that the main curve features are not changed; it's only the extreme points that are subject to changes caused by charges at the boundaries, which is evidenced by the sharp spikes accounting for short probes getting smoothed out. The signals of long probes (Epov, Martakov et al, 1999) are practically independent from conductive solution in the borehole. In practice, the commonly used diameter of a hole is $0.124 \mathrm{~m}$, which dehotes its lesser impact.

\section{High Performance GPU-based Computing}

Parallel algorithms are developed for higher productivity in solving forward and inverse problems of electromag- netic logging in calculations on GPU [15]. Development of algorithms performed with the use of Nvidia CUDA technology. Data on efficiency of the developed parallel algorithms of modeling and inversion on GPU are analysed. The most significant information is execution time of functions on the graphic device, device/host copying times, and time to copying ratio, and calculations on the device.

Efficiency estimates of calculations on the Nvidia GeForce, Tesla graphic cards (Figure 7) were obtained. Apparently, when using GPU for parallel computations, it is possible to significantly increase productivity in comparison with identical sequential calculations on the central processor (Intel Core 2 Quad 2.4 GHz). With the use of parallel algorithms and high-performance calculations on multiprocessing devices the creation of new automated interpretation systems has been reduced to practice.

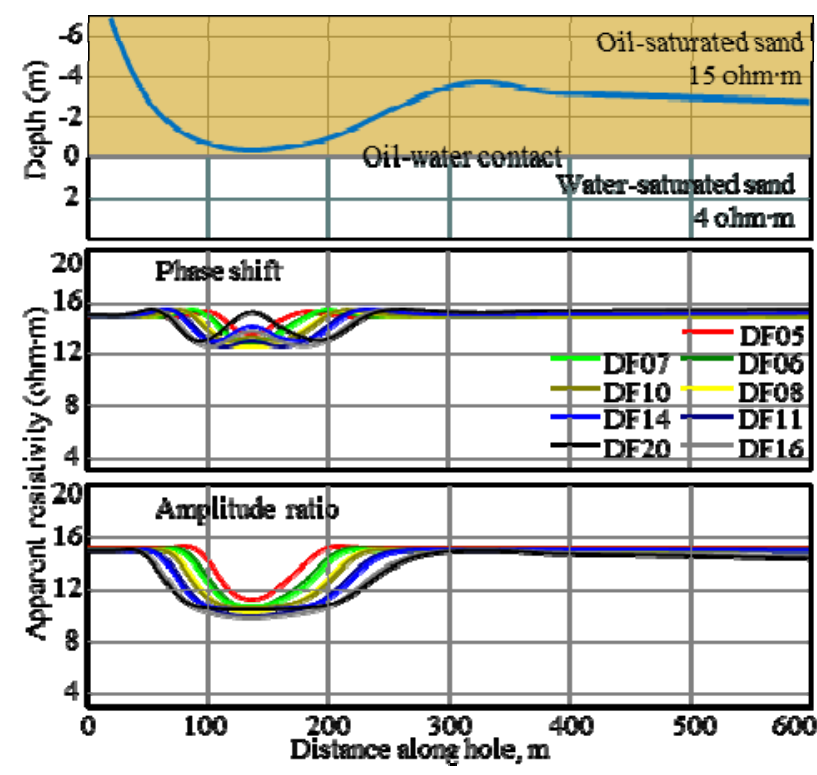

Figure 6. The model of well with horizontal completion near oil-water contact and apparent resistivity logs.

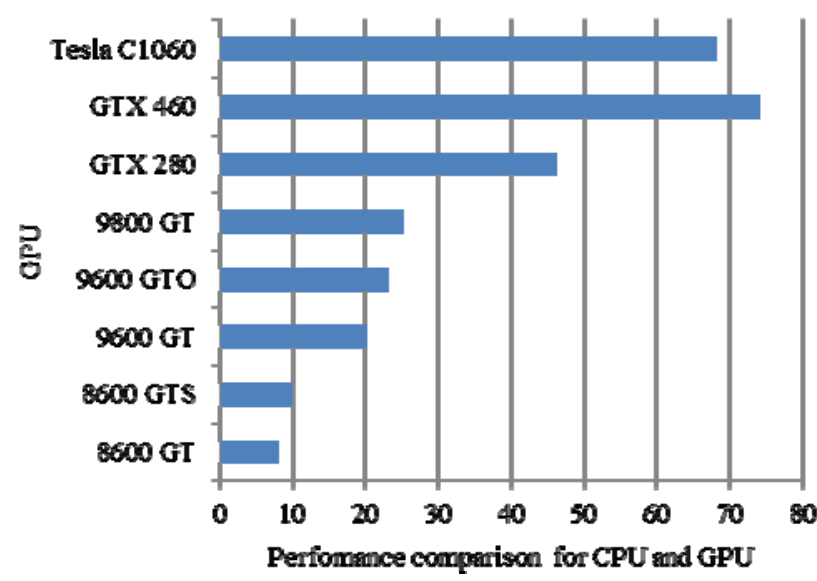

Figure 7. Efficiency increasing with GPU computing. 


\section{Conclusions}

Algorithms have been created for the solution of forward and inverse problems in numerical interpretation of electromagnetic logs in realistic models of terrigenous reservoirs penetrated by vertical, inclined or horizontal wells. There also have been developed algorithms for numerical correction of borehole rugosities and probe eccentricity effects. Effective algorithms for fast two-dimensional modeling and inversion were developed. Joint two-dimensional inversion for relative amplitude and phase characteristics measured in electromagnetic logging allows to estimate electrical conductivity and dielectric permittivity. Algorithms for modeling in inclined and horizontal wells allow to investigate signal features when approaching and crossing boundaries. High-performance calculations on GPU allow to carry out numerical interpretation of data in real time.

\section{REFERENCES}

[1] "VIKIZ Method for Logging Oil and Gas Boreholes," Ed. by Epov and Antonov. Novosibirsk: Branch "Geo" of the Publishing House of the SB RAS, 2002, p. 112.

[2] M. Epov, K. Kayurov, I. Yeltsov, K. Sukhorukova, A. Petrov, A. Sobolev and A. Vlasov, "New SKL Logging Equipment, Methods and EMF Pro Programs for Data Interpretation," Bureniei Neft, No. 2. 2010, pp. 16-19.

[3] M. I. Epov and V. N. Glinskikh, "Electromagnetic Logging: Modeling and Inversion," Novosibirsk: Publishing house Geo, 2005, p. 98.

[4] M. I. Epov, K. V. Suhorukova, M. N. Nikitenko and Ju. N. Antonov, "Application HILIS for Logging in Horizontal Wells," Russian Geology and Geophysics, Vol. 39, No. 5. 1998, pp. 649-656.

[5] M. I. Epov, M. N. Nikitenko and K. V. Suhorukova, "On the Inversion of VIKIZ Data Measured in Thinly-Laminated Formations in Inclined Borehole," Karotazhnik, No. 6, 2006, pp. 84-100.

[6] S. V. Martakov and M. I. Epov, "Forward 2D Problems of Electromagnetic Logging," Russian Geology and Geophysics, Vol. 40, No. 2, 1999, pp. 249-254.

[7] I. V. Surodina and M. I. Epov, "High-Frequency Induction Data Affected by Biopolymer-Based Drilling Fluids," Russian Geology and Geophysics, Vol. 53, No. 8, 2012, pp. 1062-1069. doi:10.1016/j.rgg.2012.06.008

[8] M. I. Epov, E. P. Shurina and O. V. Nechaev, "3D Forward Modeling of Vector Field for Induction Logging Problems," Russian Geology and Geophysics. Vol. 48, No. 9, 2007. pp. 770-774. doi:10.1016/j.rgg.2006.05.003

[9] A. D. Talalov and D. S. Daev, "Structural Mechanism of Frequency Dispersion of Electric Properties of Heterogeneous Rocks," Izvestiya Physics of the Solid Earth, Vol. 32, No. 8, 1996. pp. 683-691.

[10] B. I. Anderson, T. D. Barber, M. G. Luling, J. Rasmus, P. N. Sen, J. R. Tabanou and S. M, "Haugland Observations of Large Dielectric Effects on LWD Propagation-Resistivity Logs," SPWLA 48th Annual papers, 2007.

[11] K. V. Suhorukova, M. N. Nikitenko and A. N. Petrov, "Numerical Interpretation of VIKIZ and VEMKZ Responses in Waters of Lake Teletskoye," Karotazhnik, No. 5, 2007, pp. 30-38.

[12] A. I. Gubina, G. Z. Giniatov and I. N. Gulanov, "Hole wall Trough Influence on Logging Data," Geologiya Neftii Gaza, No. 11. 1997. pp. 38-42.

[13] M. G. Zykina and V. G. Mamjashev, "VIKIZ Log Features in Horizontal Well," Proceedings of International conference for geophysicists and geologists, Tyumen, December 3-7, 2007. CD.

[14] M. I. Epov, C. V. Suhorukova, M. N. Nikitenko, A. A. Gorbatenko and V. S. Arzhantsev, "Electromagnetic Sounding in Deviated and Horizontal Wells: Mathematical Modeling and Real Data Interpretation," SPE Russian Oil \& Gas Exploration \& Production Technical Conference \& Exhibition, Moscow, Russia 16-18th October 2012, Paper 162034-MS, p. 18.

[15] V. N. Glinskikh, M. I. Epov and I. B. Labutin, "EM Logging Diagram Modeling with Graphic Processors," Computational technologies, Vol. 13, No. 6, 2008, pp. 50-60. 